\title{
¿Pueden los Perros Domésticos (Canis lupus familiaris) Reconocer Expresiones Emocionales Humanas? una Revisión Grítica de las Evidencias
}

\author{
JULIA HELBLING ${ }^{1}$, GABRIELA BARRERA ${ }^{2}$ Y MARIANA BENTOSELA $^{1}$ \\ ICOC (Grupo de Investigación del Comportamiento en Cánidos) \\ ${ }^{1}$ Instituto de Investigaciones Médicas (IDIM) CONICET-UBA \\ ${ }^{2}$ Instituto de Ciencias Veterinarias del Litoral (ICiVet-Litoral) UNL-CONICET
}

\begin{abstract}
Las expresiones emocionales tienen un papel fundamental en las interacciones de especies sociales, brindando información acerca del emisor, las características e importancia del estímulo o situación que desencadena tales interacciones, y también funcionan regulando los vínculos sociales. Los perros poseen notorias capacidades comunicativas hacia los humanos por lo que resulta interesante estudiar si estas habilidades se extienden al reconocimiento de las expresiones emocionales. Se han realizado diversas investigaciones acerca del reconocimiento de expresiones emocionales humanas en perros exponiéndolos a una situación representada por personas durante la evaluación, así como mediante el uso de imágenes, videos y grabaciones. El análisis de las evidencias permite concluir que los perros podrían distinguir entre distintas emociones humanas ya sea en presencia de modelos en vivo o mediante la observación de imágenes y modulan su conducta conforme esta discriminación, así como también pueden utilizar esta información como clave en la resolución de problemas. Sin embargo, aún no están claros los mecanismos que subyacen a estas habilidades.
\end{abstract}

Palabras-clave: Expresiones emocionales; claves visuales; claves verbales; relación perro-humano; comunicación.

Emotional expressions play a critical role in the interactions among individuals, particularly, in social species. They provide information about the sender and the characteristics and importance of the triggering stimulus or situation, also regulating social ties. Dogs have notorious communication skills towards humans, which makes the study of their ability to recognize human emotional expressions an interesting endeavor. There have been several studies in which dogs have been tested using real people in an experimental situation as well as images, video and audio recordings of people's expressions. The evidence suggests that dogs can discriminate between different human emotions either in the presence of live models or by looking at images. This type of information has been shown to modulate their behavior and to be used as cues to solve problems. However, the mechanisms underlying these abilities are not fully understood yet.

Keywords: Emotional expressions; visual cues; verbal cues; human-dog relationship; communication.

Los animales extraen permanentemente información de su ambiente para tomar decisiones, por ejemplo dónde forrajear, qué comer, qué pareja elegir, cómo evitar predadores, dónde hallar un refugio adecuado, etc. Cuanto mayor es la complejidad de la estructura social de la especie, más cantidad de esa valiosa información proviene de los otros individuos (Bandura, 1977).

En este sentido, las expresiones emocionales juegan un papel fundamental en las interacciones de especies sociales y son parte del vasto mundo de la comunicación gestual. Ellas brindan información no sólo acerca del estado interno del emisor, sino también acerca de las características e importancia del estímulo o situación que las desencadena. Pueden mostrar que una situación es peligrosa o dolorosa y, de ese modo los otros se preparan para una respuesta de lucha o huida, o también pueden indicar alegría o sorpresa desencadenando en los demás respuestas de acercamiento al estímulo (Ekman, 1993; Stetina et al., 2011).

Correspondencia a: Mariana Bentosela, Grupo ICOC (Investigación del Comportamiento en Cánidos), Instituto de Investigaciones Médicas (IDIM) CONICET-UBA, Combatientes de Malvinas 3150 (1426). Buenos Aires, Argentina. E-mail: marianabentosela@gmail.com 
También la expresión de emociones funciona regulando los vínculos sociales. Si ante determinado comportamiento el otro reacciona con una emoción positiva, como la alegría, esto probablemente refuerce nuestra conducta y aumente su probabilidad de repetirla en el futuro. Si por el contrario la reacción es negativa, como de enojo o tristeza, tenderemos a evitar ese comportamiento en el futuro y probablemente desarrollemos respuestas de consuelo o protección frente a esas claves (Izard, 1990).

Para poder utilizar la extensa información que nos brindan las expresiones emocionales, debemos ser capaces de detectarlas y discriminarlas unas de otras. Cuando hablamos de expresiones emocionales nos referimos a un compuesto estimular amplio y complejo, que incluye todas las modalidades sensoriales y que, a veces, depende de claves sutiles que duran tan solo unos breves instantes. En humanos hay un predominio de lo visual, aunque también, gracias al lenguaje e incluso a otras vocalizaciones, lo verbal ocupa un rol relevante. El hecho de que las emociones involucren un conjunto de claves multisensoriales, que con frecuencia son sutiles y de corta duración, implica que la demanda cognitiva para la detección de emociones sea elevada (Graham \& LaBar, 2012).

Diversos estudios han demostrado que algunas expresiones emocionales en humanos son comunes en diversas culturas. Un estudio clásico sobre este tema que evaluó expresiones de felicidad, miedo, sorpresa, tristeza, ira y asco, en cinco culturas letradas, reveló que la identificación de dichas emociones fue la misma en la mayoría de los sujetos encuestados. Este hallazgo sugeriría una cierta universalidad en las expresiones faciales humanas (Ekman, 1971). Sin embargo, según Russell (1994), existen diferencias culturales, por ejemplo, mientras que en algunas regiones del planeta no mirar a una persona a la cara se considera de mala educación, en otras mirar a los ojos es un gesto desafiante.

En la naturaleza abundan las especies sociales y en algunos casos los intercambios no se limitan a conespecíficos, sino también a miembros de distintas especies que, por alguna razón, están sometidos a una interacción sostenida. En estos casos no basta con que el individuo detecte y reconozca las claves emocionales intraespecíficas, sino que, además puede ser ventajoso, ser capaz de codificar la forma en la que la otra especie manifiesta sus emociones. Uno de los casos más llamativos es el que se produce entre el perro doméstico (Canis lupus familiaris) y el hombre. En este sentido, los perros han mostrado tener notables capacidades comunicativas con los humanos (Hare \& Tomasello, 1999; Udell, Dorey, Wynne, 2010) tales como: la utilización de diversas claves humanas, como el señalamiento (Elgier \& Bentosela, 2009); la emisión de señales comunicativas, como la mirada, cuando hay un reforzador a la vista pero inaccesible (Miklósi et al., 2003); y también la habilidad de extraer información contenida en los mensajes complejos e integrar en el desempeño de las instrucciones dadas por las personas (Ramos \& Ades, 2012). De este modo, resulta de gran relevancia estudiar si estas habilidades se extienden al reconocimiento de las expresiones emocionales.

El objetivo del presente manuscrito es realizar una revisión exhaustiva y critica de los trabajos existentes hasta el momento en la literatura sobre el reconocimiento de emociones humanas en perros, haciendo especial énfasis en las metodologías utilizadas. Primero, describiremos las razones que vuelven a los perros candidatos idóneos para poseer tal habilidad. Enseguida, realizaremos una breve reseña del reconocimiento humano de las expresiones emocionales de los perros. Finalmente, en la conclusión recapitularemos los resultados alcanzados así como los posibles caminos a seguir, poniendo énfasis en el valor que esta habilidad puede tener en el vínculo entre ambas especies y se discutirán los mecanismos involucrados en esta capacidad.

\section{${ }_{\mathrm{d}}$ Pueden los perros reconocer emociones humanas? Un análisis de las evidencias experimentales}

Son innumerables los ejemplos anecdóticos que aseguran que los perros tienen la capacidad de reconocer nuestras emociones y actuar en consecuencia. La famosa frase "sabe cuando estoy triste y me ayuda o me consuela" es sumamente conocida por muchos dueños y admiradores de estos animales. Sin embargo, el ritmo de las investigaciones científicas acerca del tema no ha acompañado al del conocimiento popular. Las evidencias empíricas del reconocimiento de emociones son aún escasas.

Probablemente la primera pregunta que surge es por qué los perros serían candidatos idóneos para detectar nuestras claves emocionales. Existen varios factores relevantes para ello. Si por un lado las evidencias genéticas indican que el proceso de domesticación lleva alrededor de 15.000 años, periodo durante el cual los perros han estado 
compartiendo el mismo ambiente con los humanos (Axelsson et al., 2013; Davis \& Valla, 1978; Vila et al., 1997; Lindblad-Toh et al., 2005; Pang et al., 2009). Por este motivo, ambas especies habrían desarrollado capacidades para comprender claves comunicativas que facilitaran la convivencia (Hare et al., 2010). Si bien el perro está más alejado filogenéticamente del hombre que otras especies - por ejemplo los chimpancés -, pero el estrecho contacto cotidiano, incluso desde el nacimiento, así como el largo e intenso proceso de domesticación al que fue sometido lo convierte en un modelo óptimo para estudiar habilidades comunicativas interespecíficas (Miklósi, 2009). Además provienen de los lobos que tienen una compleja estructura social y un amplio repertorio comunicativo intraespecífico (Miklósi, 2009).

Los trabajos que evalúan la capacidad de los perros de reconocer y discriminar expresiones emocionales humanas podrían agruparse en dos categorías. En primer lugar, algunas investigaciones exploraron esta habilidad exponiendo a los animales a una situación representada por personas en el momento de la evaluación (Vas, Topál, Gas, Miklósi, \& Csányi, 2005). Este tipo de trabajo tiene la ventaja de poseer una enorme validez ecológica en tanto replica, con una gran similitud, situaciones que el perro ha probablemente experimentado repetidas veces en su vida cotidiana. No obstante posee la desventaja de que involucra un numeroso compuesto estimular en el cual es imposible distinguir cuales son las señales más salientes que utiliza el perro para discriminar una determinada emoción. En segundo lugar, algunos estudios presentan ciertas claves en forma aislada, ya sea porque en la representación de las personas eliminan una modalidad sensorial, por ejemplo las claves vocales (Marshall-Pescini, Passalacqua, Miletto Petrazzini, Valsecchi, \& Prato-Previde, 2012), o porque presentan imágenes o grabaciones en que solo hay un único tipo de información (Nagasawa, Murai, Mogi, \& Kikusui, 2011). Si bien estos estudios aportan un mayor nivel de control experimental, muchas veces la dificultad aquí radica en que el perro sea capaz de prestar atención a un estímulo artificial y que la falta de respuesta no pueda atribuirse a una inadecuada codificación sensorial de la información, por ejemplo al presentar señales en dos dimensiones como las fotos. Ambos tipos de estudios son sin duda complementarios y aportan información valiosa para el entendimiento de las habilidades sociales de los perros.

\section{Estudios multidimensionales}

Dentro del primer grupo antes mencionado, Vas et al. (2005) investigaron cómo los perros son capaces de modificar sus conductas en respuesta a las actitudes diferenciales de una persona. Con este fin éstos animales fueron expuestos a acercamientos alternativos de una mujer desconocida que mostraba en un caso claras señales amistosas mientras que en el otro se comportaba de modo amenazante. Las observaciones consistieron en dos episodios: la desconocida se acercaba al perro caminando a velocidad normal mientras que le hablaba y finalmente lo acariciaba suavemente (acercamiento amistoso) o se movía lenta y vacilantemente con el torso inclinado mirando fijamente a los ojos del perro sin ninguna comunicación verbal (acercamiento amenazante). Cuando la persona se acercó amigablemente casi todos los perros reaccionaron de manera "positiva" o "pasiva" en contraste con el acercamiento amenazante en el cual la mayoría respondió evitando la interacción con el extraño, alejándose, desviando la mirada y emitiendo vocalizaciones. Más aun, utilizando el mismo procedimiento se hallaron diferencias significativas entre tres grupos de razas. Los pastores belgas mostraron mayor evitación o signos de agresividad cuando los acercamientos fueron amenazantes. Tanto los retrievers como los perros de trineo fueron menos propensos a evitar al extraño. No se encontraron diferencias en cuanto al sexo, pero las comparaciones entre razas sugieren que la cría selectiva pudo haber influenciado la sensibilidad de los perros a las claves sociales humanas de tipo atemorizantes si bien no queda claro como pudo darse este proceso en las razas aquí evaluadas.

También interesados en evaluar si los perros son capaces de diferenciar actitudes positivas y negativas en las personas, Nitzschner, Melis, Kaminski y Tomasello (2012) evaluaron un componente clave en las interacciones sociales de muchas especies animales: la reputación. Ésta podría considerarse como la atribución de una característica estable de un individuo que permite predecir su comportamiento en ciertas situaciones sociales, y puede ser elaborada a partir de dos fuentes principales: el resultado de la experiencia directa en repetidas ocasiones con el individuo, o en forma indirecta, a partir de la observación de las acciones que un individuo tiene con un tercero (Russell, Call \& Dunbar, 2008).

Para investigar la habilidad de asignar reputación se realizaron dos experimentos. En el primero, los sujetos tenían interacción directa con un humano 
"agradable" (que les hablaba, jugaba con ellos y los acariciaba) o con un experimentador "indiferente" que los ignoraba completamente. Luego el sujeto podía elegir entre ambos. Los resultados mostraron que los perros permanecían más tiempo cerca del humano "agradable". En un segundo experimento debían elaborar la reputación en forma indirecta. Para ello los perros observaron a un humano "agradable" o "indiferente" en interacción con otro perro detrás de un cubículo plegable de Plexiglás. También se realizaron pruebas en el cual el sujeto podía elegir entre ambos experimentadores. Hallaron que los perros durante la observación de las interacciones se comportaron de manera diferente en función de si era el experimentador agradable o indiferente, lo cual sugiere que distinguen las diferentes actitudes de las personas. Sin embargo no mostraron en la prueba de elección posterior una preferencia por ninguno de ellos, tal como había ocurrido cuando los perros tenían una experiencia directa con los humanos (Nitzschner, Melis, Kaminski \& Tomasello, 2012). Estos últimos resultados contrastan con estudios previos que mostraron que los perros eran capaces de realizar una inferencia de reputación indirecta pero en una situación de "pedir" comida (Kundey et al., 2011; Marshall-Pescini et al., 2012). En el futuro se podrían investigar los factores que limitan la capacidad de los perros en este dominio cuando el reforzador sea social en lugar de comida, por ejemplo, probando, así, este método con otros roles, o con interacciones entre humanos, como ejemplo experimentador-dueño, más que interacciones entre perros y humanos. Sin embargo a los fines de esta revisión es relevante destacar que, en este estudio, en ambos experimentos se demostró que los perros pueden distinguir entre los diferentes tipos de actitudes y expresiones humanas que dan lugar a diferentes tipos de interacciones.

Según Vitulli (2006), un aspecto del vínculo entre personas y perros es el hecho de que éstos parecen estar sincronizados empáticamente con las emociones humanas. La empatía abarca una serie de fenómenos que involucran la representación cognitiva de las emociones ajenas, su respuesta, por su vez, se caracteriza por estar orientada hacia otro en vez de a sí mismo, por lo tanto un indicador de empatía puede ser ofrecer comodidad o ayuda en respuesta a la tristeza de otro. Custance y Mayer (2012) tomaron como modelo un protocolo experimental utilizado en infantes humanos, para investigar la empatía con perros. Estas autoras combinaron dos factores para evaluar la discriminación entre diferentes estímulos: llanto vs tarareo y dueño vs desconocido, para formar cuatro situaciones diferentes a las que fueron expuestos los perros. Cada condición de llanto o tarareo era precedido por 2 min durante el cual el propietario y el desconocido hablaban. La condición de tarareo se incluyó para ver si los acercamientos de los perros estaban motivados por la curiosidad, y también se evaluó el tono emocional de los acercamientos durante las diferentes condiciones (es decir, llorando y tarareando). Así se supuso que si los perros estaban exhibiendo tristeza contagiosa o empatía, sería esperable que no se comporten de manera juguetona o alerta. Se observó que los perros se acercaron a la persona, independientemente de si era su dueño o un extraño, con más frecuencia cuando ésta simulaba llorar que cuando hablaba o tarareaba. En estos acercamientos los perros tomaron una postura calma o sumisa. De esta manera, las autoras sostienen que es posible que la respuesta de los perros al llanto sea por contagio emocional, porque la situación les despertara tristeza y, así, buscaran obtener comodidad para sí mismos más que consuelo para los humanos. Sin embargo, si los enfoques de los perros durante la condición de llanto estaban motivados exclusivamente por comodidad egoísta, uno podría esperar que sea más probable que se acercaran a su fuente habitual de comodidad, es decir, su propietario, en lugar del desconocido. No obstante, no se encontró tal preferencia.

Por otro lado, no se puede hablar de verdadera empatía ya que no hay evidencia de que exista una cierta comprensión de la perspectiva mental del llanto de los seres humanos. Una posible explicación alternativa, más parsimoniosa, del comportamiento exhibido es que los perros hayan recibido en su vida cotidiana reforzamiento positivo por acercarse a personas que lloran. Cualquier perro del hogar que se acerca a un miembro de la familia que está llorando es probable que sea positivamente reforzado mediante respuestas afectivas lo que aumentaría las posibilidades de que en el futuro se acerque a personas que exhiban tales claves conductuales. Por otro lado desde un punto de vista metodológico, otra posibilidad es que en la expresión de llanto, las personas colocaban sus manos sobre sus caras. Esa actitud pudo despertar curiosidad en los perros o incluso incertidumbre, aún más que el tarareo. Parece además llamativo entender por qué las autoras suponían que el tarareo despertaría curiosidad en los perros y serían un adecuado grupo control. Las mismas autoras exponen que hubiese sido mejor comparar la risa con el llanto para ver las respuestas diferenciales 
de los perros. De estas cuestiones se desprende que el estudio de Custance y Mayer (2012), si bien representa un intento interesante, no permite extraer conclusiones claras y requeriría de una modificación del diseño experimental utilizado.

En síntesis, de los estudios multidimensionales analizados se podría concluir que los perros pueden distinguir entre actitudes positivas y negativas en los humanos basándose en la observación de situaciones en vivo. En éstas los perros parecen reconocer un conjunto de claves conductuales y sensoriales que modulan su comportamiento de acuerdo a la valencia de tales situaciones. Los estudios realizados hasta el momento no permiten concluir si este cambio conductual está asociado a procesos de empatía de los perros hacia las personas o al hecho de que durante su ontogenia ellos han asociado esas claves indicadoras de emociones con diferentes consecuencias para sí, tanto apetitivas como aversivas, y en función de ello modifican sus respuestas.

Por otro lado, el uso de expresiones emocionales no se limita sólo a regular los vínculos sociales sino que también provee claves para resolver situaciones problemáticas que permitan el acceso a diferentes recursos.

Las expresiones emocionales como claves comunicativas en la resolución de problemas

Una tarea de resolución de problemas implica una situación en que no se puede llegar al objetivo de manera directa por lo que se deben implementar conductas que estén por fuera del repertorio comportamental habitual (Fragaszy \& Liu, 2012). En el curso del proceso de domesticación y durante la estrecha convivencia en la ontogenia, los perros aprendieron a utilizar la información social proveniente de las personas para resolver problemas y modificar su comportamiento. En este sentido, ellos utilizan con frecuencia respuestas sociales interespecíficas para acceder a reforzadores que no están a su alcance (Bentosela et al., 2008; Udell et al., 2010).

En esta línea Buttelmann y Tomasello (2013), basándose en el estudios en bebes realizado por Repacholi (1998), investigaron el uso de expresiones comunicativas humanas como claves para los perros, es decir, si pueden utilizarlas para hallar comida. En este trabajo, una persona reaccionaba emocionalmente (feliz, neutral o con disgusto) a los contenidos ocultos en dos cajas y después se permitía al perro elegir una de las cajas. Las expresiones emocionales de felicidad y disgusto se basaron en las descripciones de Ekman y Friesen (1976). Para la expresión neutral el experimentador dejó los ojos abiertos, la boca cerrada y los músculos faciales relajados. Se agregaron vocalizaciones para las expresiones de felicidad y disgusto, no así para la condición neutral. Los autores no ofrecieron una explicación del porqué de esta diferenciación, la cual podría representar un error metodológico que podría corregirse en futuras investigaciones que utilicen este modelo.

Así, se evaluaron cuatro razas de perros en el laboratorio y una al aire libre. Los perros evaluados en el laboratorio discriminaron mejor entre emociones muy distintas (feliz-disgustado), pero eligieron al azar cuando las dos emociones eran menos claras (feliz-neutral). Por otro lado, la raza evaluada al aire libre pudo discriminar las dos condiciones. Sin embargo, esta diferencia pudo deberse a que las razas fueron evaluadas, sin justificación por parte de los autores, en lugares diferentes y esto podría explicar las diferencias en el desempeño. Por esta razón no es posible concluir que haya diferencias de razas en esta habilidad.

Los perros pueden solicitar a las personas no sólo objetos concretos sino que también pueden recurrir a ellas en busca de información cuando se encuentran en una situación de incertidumbre. Merola, Prato-Previde y Marshall-Pescini (2011) utilizaron el paradigma de referencia social entendida como la búsqueda de información de otro individuo para comprender y guiar la acción. Este paradigma tiene dos componentes: la búsqueda de información y la regulación del comportamiento. La referencia social al igual que todos los procesos de aprendizaje social, permite al individuo evitar costosos errores asociados con el aprendizaje por ensayo y error (Russell, Bard, \& Adamson, 1997). Muchos estudios demostraron la presencia de referencia social en bebés y niños: éstos miran al informante y cambian su comportamiento de acuerdo al mensaje emocional recibido (Kim, Walden, Knieps, 2010; Mumme, Fernald, \& Herrera, 1966; Sorce, Emde, Campos, \& Klinnert, 1985).

En este estudio los perros adultos fueron evaluados en un paradigma de referencia social que incluye a su dueño y a un objeto potencialmente atemorizador. El estímulo utilizado fue un ventilador eléctrico con cintas plásticas atadas que estaba en el otro extremo de la habitación funcionando. El dueño tenía primero una expresión neutral y luego manifestaba una respuesta positiva o negativa hacia el objeto de intensidad creciente. La mayoría de los perros $(83 \%)$ miraron referencialmente al dueño 
después de ver el objeto extraño, por lo que, frente a un estímulo ambiguo, parecen buscar información en el humano. Además, en lo referente a la regulación del comportamiento, los perros del grupo negativo - en comparación con el grupo positivo - estuvieron quietos por más tiempo e interactuaron más con el dueño en las fases en que la expresión emocional era más exagerada. Esta influencia en el comportamiento podría deberse a un proceso de condicionamiento observacional, acercándose al ventilador los del grupo positivo y alejándose los del negativo, ambos reflejando el comportamiento de su dueño. En un estudio posterior Merola, Prato-Previde y Marshall-Pescini (2012) mostraron nuevamente que los perros fueron capaces de discriminar las reacciones emocionales de su dueño y de un extraño y modificaron su comportamiento en función de esas reacciones, pero respondieron más ante su dueño que ante el extraño. Cuando el informante era el dueño los perros en el grupo positivo se acercaron hasta el ventilador más rápidamente y los perros del grupo negativo tardaron más. Cuando el mensaje era negativo los perros miraron más a la persona sentada cuando se trataba de su dueño que cuando era un extraño, por lo que su comportamiento parece ser selectivo y dependiente de la relación que mantienen con el informante. Dada la historia previa con el dueño es posible que a los perros les resulte más fácil discriminar las expresiones emocionales de una persona familiar que de una desconocida.

Estos estudios demostrarían que los perros utilizan la mirada no sólo para pedir objetos o comida sino también para monitorear la reacción de una persona (familiar o desconocida) hacia un objeto incierto. Tomar información emocional acerca de un estímulo novedoso que no puede predecirse si será bueno o malo podría significar de un enorme valor para los perros, y de esta forma podrían evitarse posibles peligros que implicarían altos costos para la supervivencia y el bienestar del animal.

Por último, otra forma de evaluar la detección de expresiones emocionales positivas en las personas es a través del efecto que producen en diversas tareas el uso de claves ostensivas. Éstas tales como la entonación y las verbalizaciones positivas así como la alternancia de mirada entre un receptor y el objeto de interés sirven no solo para incrementar la atención del receptor sino también para definir un determinado contexto como comunicativo. Conforme aportan algunos autores son un modo de establecer un grado de intencionalidad en la comunicación (Topál, Gergely, Erdöhegyi, Csibra, \& Miklósi, 2009).
Por ejemplo, en un estudio realizado por Topál et al. (2009) se indujo a los perros mediante claves comunicativas a perseverar en un error buscando objetos escondidos en un sitio donde no había nada. En la primera fase, se realizaron cuatro ensayos en que el perro recogía un juguete repetidamente detrás de la pantalla $\mathrm{A}$, seguido de tres ensayos en el que el experimentador escondió el objeto repetidamente detrás de la pantalla alternativa $B$. Luego se variaron las condiciones en función de las claves administradas: una condición socio-comunicativa en la cual el experimentador atrae la atención del perro mediante claves ostensivas y le indica donde está el objeto, una condición no-comunicativa el experimentador realiza las mismas acciones dándole la espalda al perro, sin establecer contacto con él y, finalmente, una condición no-social en la que el objeto era manipulado mediante un hilo transparente sin que se viera la manipulación del experimentador y sin ningún tipo de señales comunicativas. Los perros cometieron mayores errores perseverando en la búsqueda detrás de la pantalla $\mathrm{A}$ en la condición socio-comunicativa que en las otras dos condiciones restantes. Estos resultados indican que al igual que los bebés humanos (Topál et al., 2008) el contexto comunicativo induce a los perros a realizar una búsqueda perseverante y errónea de objetos que previamente fueron ocultados en una posición (A) aún cuando pudieron ver que el objeto en realidad se ocultaba en una posición diferente (B).

En esta misma línea, en un estudio realizado por Prato-Previde, Marshall-Pescini y Valsecchi (2008) se halló que, ante dos cantidades diferentes de comida los perros generalmente elegían la de mayor cantidad, pero cambiaban su preferencia cuando el dueño mostraba interés por la menor cantidad de alimento, acercándose a ese plato, y emitiendo expresiones verbales positivas.

Para entender los mecanismos responsables de este fenómeno, Marshall-Pescini et al. (2012) presentaron a los sujetos dos platos con cantidades diferentes o iguales de comida, quedando así tres condiciones: influencia neutral (elección entre dos cantidades con el demostrador mostrando interés por uno u otro), no influencia (los perros pueden elegir sin influencia, esta condición fue idéntica para todos los grupos) y de influencia contraproducente (la elección entre las cantidades grande y pequeña con un demostrador que muestra interés por la pequeña). Los resultados mostraron que los perros discriminan claves emocionales humanas tales como el uso de un tono de voz positivo y la mirada. Además se 
encontró que el uso combinado de estas claves ejerce una influencia mayor en su comportamiento (los animales actuaron en contra de su preferencia) que cuando se presentaron de forma aislada. Sin embargo, hallaron que la forma más poderosa para llamar la atención no eran las claves comunicativas (solas o combinadas) sino más bien la acción de llevar la mano a la boca con o sin contacto con los alimentos. Esto demostraría que las señales humanas pueden influir fuertemente en los dos aspectos del comportamiento de los perros pero no necesariamente lo hacen en paralelo.

En síntesis, los estudios de este apartado muestran que los perros son capaces de discriminar diferentes expresiones emocionales incluidas el tono de voz y la dirección de la mirada y utilizan esta información para resolver diversos problemas. Resulta interesante que las expresiones emocionales le permiten no solo acceder a refuerzos valiosos, como la comida, sino que también frente a una situación ambigua los perros utilizan las expresiones emocionales humanas para guiar su conducta. Como mencionáramos previamente, estos trabajos están basados en modelos en vivo que representan las diferentes expresiones emocionales a través de un compuesto estimular. A continuación se discutirán los trabajos que han intentado evaluar la capacidad de los perros de discriminar claves emocionales humanas a través de una única modalidad sensorial, utilizando estímulos visuales.

\section{Estudios con estímulos visuales}

La mayoría de los estudios en que se presentan claves con una sola modalidad sensorial utilizan imágenes visuales como estímulos para presentar claves faciales. Diversas evidencias muestran que las señales visuales son significativas para cánidos domésticos en las interacciones entre sus congéneres e individuos de otras especies (Gaunet \& Deputte, 2011; Hare \& Tomasello, 1999; Virányi, Topál, Gácsi, Miklósi, \& Csányi, 2004; Virányi, Gácsi, Kubinyi et al. 2008).

Un requisito para la detección de expresiones emocionales podría ser que los perros sean capaces de codificar rostros, tanto de sus congéneres como de humanos, y si pueden agruparlos dentro de una misma categoría sólo partiendo de claves visuales. En la mayoría de las interacciones sociales un animal tiene que determinar si el otro animal pertenece a su propia especie. Autier-Dérian, Deputte, ChalvetMonfray, Coulon y Mounier (2013) se preguntaron si esta habilidad estaba presente en los perros domésticos que se caracterizan por una inmensa diversidad fenotípica. Buscando responder a este interrogante realizaron un protocolo experimental en que debían elegir una imagen entre dos pantallas de computadora, a partir de un aprendizaje discriminativo en el cual el rostro del perro estaba asociado a la comida y el rostro de otra especie a la ausencia de comida. Luego se evaluó si los perros podían transferir la respuesta correcta a estímulos no entrenados, de modo de evidenciar la formación de la categoría y no solo la respuesta a estímulos aprendidos. Se presentaron pares de rostros de varios tipos de razas de perros y otras especies que no habían sido presentadas previamente (rostro de perro con rostro de vaca). Los resultados mostraron que los sujetos fueron capaces de agrupar todas las imágenes de perros dentro de la misma categoría, basándose solamente en las imágenes visuales de las cabezas. Un siguiente paso podría ser determinar cuáles son las características más salientes de los rostros de los perros, en las que se basan para hacer dicha discriminación y categorización.

En el mismo sentido, otro requisito para la detección de emociones a partir de claves faciales podría ser la detección de rostros familiares en comparación con rostros nuevos lo que indicaría que poseen la capacidad de procesar los rasgos pertenecientes a la cara de otro perro o de una persona. En este sentido, Racca et al. (2010) realizaron un estudio en el que utilizaron el paradigma de mirada diferencial, según el cual si el sujeto puede discriminar entre lo familiar y lo novedoso, prestará mayor atención al estímulo novedoso. En la primera fase de familiarización les presentaban a los perros una imagen en la pantalla, seguida de otra fase donde les presentaban la imagen con la que fueron familiarizados anteriormente en conjunto con una imagen novedosa. Los perros pudieron discriminar los rostros familiares tanto de perros como de humanos.

En síntesis estos estudios muestran que los perros pueden procesar los aspectos relevantes de los rostros tanto de otros perros como de personas. La siguiente pregunta es si pueden discriminar ciertas expresiones faciales a partir de la observación exclusiva de las caras. Para responder a este interrogante, Nagasawa et al. (2011) llevaron a cabo una tarea de discriminación entre dos opciones, rostros humanos sonrientes asociados con comida y rostros humanos inexpresivos asociados a la ausencia de comida, mediante la presentación de fotografías. Para ello primero evaluaron esta habilidad utilizando imágenes del dueño y luego fotografías de personas 
desconocidas del mismo y de distinto género al del dueño de modo de establecer el grado de generalización que los perros tienen en esta habilidad. Los resultados mostraron que los animales eligen significativamente más el rostro sonriente en las pruebas donde se presentaban imágenes del dueño e imágenes de personas desconocidas del mismo sexo que el dueño, no encontrándose diferencias significativas en el caso de personas desconocidas de distinto sexo. Según estos autores una posible explicación podría ser que el perro hiciera una generalización dependiendo del género. El perro toma mucha información del dueño a diario y esto puede hacer que tenga peor rendimiento con personas de distinto género a su dueño. Sin embargo no fueron capaces de discriminar la expresión de enojo, probablemente porque en sus vidas cotidianas estuvieron menos expuestos a ella. Estos resultados muestran, por un lado, que los animales discriminan a partir de claves faciales al menos ciertas expresiones emocionales. Sin embargo, la experiencia con dichos estímulos parece ser fundamental para un desempeño exitoso.

Por otro lado, es importante rescatar que si bien los animales fueron entrenados para realizar la discriminación y el nivel de generalización de los resultados fue limitado, la dificultad de la tarea era elevada. Los estímulos en el experimento fueron fotografias en dos dimensiones y el contexto pudo ser insuficiente para discriminar rostros humanos que normalmente se presentan en tres dimensiones. En futuros estudios sería interesante determinar qué partes de la cara están involucradas en el procesamiento visual que hacen los perros para discriminar expresiones faciales humanas, y conocer si pueden categorizar estas expresiones.

Posteriormente, se evaluó la misma habilidad utilizando claves visuales pero usando una medida dependiente diferente que podría tener un mayor nivel de sensibilidad que la del estudio previo. Racca, Guo, Meints y Mills (2012) estudiaron si existe algún sesgo de mirada cuando los perros presencian imágenes de rostros expresivos. El procesamiento de las emociones está sujeto a la lateralización del cerebro. En perros domésticos se halló un predominio hemisférico derecho (giro de la cabeza preferentemente hacia la izquierda) a estímulos amenazantes y estímulos emocionalmente intensos (Siniscalchi, Quaranta, \& Rogers, 2008; Siniscalchi, Sasso, Pepe, Vallortigara, \& Quaranta, 2010). La preferencia por uno u otro campo visual refleja el uso de uno de los hemisferios en el procesamiento de las expresiones faciales emocionales. Abordando este interrogante realizaron un experimento en que los sujetos observaron en una pantalla imágenes de rostros humanos y de perros con diferentes expresiones emocionales: negativa (amenaza), neutral y positiva (amistosa). Se cubrió un espacio horizontal amplio con imágenes de objetos cuasi-simétricos a fin de obtener movimientos oculares laterales. Los perros presentaron un sesgo de mirada a la izquierda cuando veían un rostro con expresión negativa y hacia la derecha cuando observaban un rostro con expresión positiva, mostrando que pueden discriminar las emociones con diferente valencia. Estos resultados replican y extienden los hallazgos de Nagasawa et al. (2011) mostrando que los animales detectan expresiones emocionales tanto positivas como negativas, a partir de claves faciales.

Recapitulando, de las investigaciones revisadas anteriormente podemos concluir que los perros serían capaces de hacer discriminaciones de las expresiones emocionales basándose en claves más sutiles como la utilización de imágenes en dos dimensiones. Sin embargo, la tarea requiere una demanda cognitiva mayor puesto que la discriminación de rostros normalmente se realiza en tres dimensiones, por lo que los sujetos podrían no disponer de información suficiente. Además se observó que la distinción entre expresiones emocionales de diferente valencia se realiza con mayor facilidad cuando se trata del dueño o de personas del mismo sexo que éste, lo cual confirmaría la importancia de la experiencia previa en dicha discriminación.

El conjunto de estudios, tanto multi como unidimensionales, demostraría que los perros que viven estrechamente con los humanos son capaces de reconocer al menos algunas de sus expresiones emocionales, lo que representa una importante ventaja adaptativa. Hasta aquí hemos abordado parte de la literatura existente sobre el reconocimiento de expresiones emocionales en perros. Existen también algunas investigaciones que buscaron echar luz sobre la cuestión de si los seres humanos podemos reconocer y clasificar expresiones emocionales caninas, sin embargo las evidencias todavía son escasas.

\section{¿Pueden los humanos reconocer expresiones emocionales de los perros?}

Para explorar la capacidad humana de discriminar expresiones faciales de perros, Bloom y Friedman (2013) realizaron un experimento en el cual presentaron a los sujetos fotografias del rostro de un perro - ovejero alemán -, tomadas bajo 
condiciones comportamentales que provocarán respuestas emocionales específicas. Se usaron las 6 emociones básicas descritas por Ekman y Friesen (1976): felicidad (jugar con una pelota), tristeza (reprimenda), sorpresa (muñeco que sorpresivamente salta de una caja de la que está sujeto por un resorte), asco (recibir una solución desagradable), ira (un "hombre malo" molestaba al perro) y miedo (se acercaban con un objeto para cortarle las uñas mostrándoselo y verbalizando la intención). Adicionalmente se utilizó una condición neutral (sentado tres minutos sin hacer nada). Expertos en perros clasificaron y evaluaron las fotografías y las mejor valoradas fueron usadas como estímulos para personas con y sin experiencia profesional con perros. Se examinó si las personas podían juzgar estos estados emocionales e identificar las condiciones en que se habían tomado las fotografías en base a una hoja donde estaban descriptas las distintas situaciones comportamentales utilizadas.

Se encontró que los humanos podían clasificar las emociones transmitidas y que las personas con experiencia identificaron mejor los comportamientos definidos por el contexto, es decir, pudieron identificar con más facilidad la fotografía correspondiente a cada descripción que se les entregó por escrito. Esto podría deberse a que las personas con experiencia tienen conocimiento específico de cómo un perro va a reaccionar a las condiciones dadas. Los que no poseían experiencia con perros aparentemente entendieron bien las emociones pero no identificaron el efecto que producirían las diferentes situaciones.

Este estudio constituiría un indicio de que los seres humanos serían capaces de identificar expresiones emocionales faciales en un perro, y que al igual que lo observado en los perros, la experiencia previa favorece este tipo de discriminaciones. Sin embargo, dado que se utilizó una sola raza y un solo perro como modelo sus resultados presentan una generalización limitada, por lo que se requieren futuros estudios que corroboren estos hallazgos.

En el mismo sentido, Wan, Bolger y Champagne (2012) investigaron como los humanos perciben las emociones de los perros en base a la observación de videos que se presentaron en una pantalla a sujetos con distintos niveles de experiencia con estos animales. Para ello compararon profesionales (personas que trabajaban con perros en distintas tareas), personas que hayan tenido experiencia con perros y aquellas sin experiencia. Los videos fueron clasificados previamente por expertos en dos categorías: comportamiento "alegre" o "asustado". Hallaron que todas las personas podían reconocer adecuadamente la expresión alegre mientras que el nivel de experiencia influyó en la identificación del comportamiento temeroso. La habilidad de los humanos para identificar la expresión de miedo se incrementaba conforme aumentaba su nivel de experiencia con los perros. También, las personas experimentadas tuvieron menos dificultad y mayor precisión en la tarea según informaron en cuestionarios autoevaluativos, y pudieron referir una mayor cantidad de características físicas que les informaran del estado emocional de los animales (ojos, oídos, boca / lengua, piernas / patas, cola).

Sin embargo, este trabajo presenta algunas limitaciones. Existen aspectos del comportamiento de los perros en vivo, tales como el contacto visual entre el perro y el observador y señales contextuales, que no pueden ser replicados en un video, y por lo tanto, es posible que los efectos no sean los mismos que en situaciones reales. Asimismo las categorías no son exhaustivas y no comprenden todas las respuestas emocionales posibles de miedo y alegría. Otro aspecto es que no se tomaron medidas fisiológicas de los perros en las distintas situaciones. Sin embargo pese a las limitaciones antes mencionadas, el estudio arroja datos significativos en cuanto a la percepción de emociones y cómo es influenciada por las diferencias individuales en cuanto a la experiencia previa. Más aún este efecto de la experiencia también fue observado en el reconocimiento intraespecífico de las emociones humanas (Pollak, Cicchetti, Hornung \& Reed, 2000).

\section{Conclusión}

El objetivo de este trabajo fue realizar una revisión de los estudios acerca del reconocimiento de las expresiones emocionales humanas en perros domésticos. Como vimos anteriormente, esta habilidad plantea una demanda cognitiva importante debido a que muchas veces se trata de claves muy sutiles y de breve duración a través de diferentes modalidades sensoriales. El reconocimiento de ellas podría tener consecuencias relevantes para la toma de decisiones y la regulación de los vínculos sociales, en este caso en particular entre el perro y el hombre.

De los estudios recopilados es posible concluir que los perros podrían distinguir entre actitudes humanas de diferente valencia, tanto positiva como negativa, y realizar esta discriminación en 
presencia de modelos en vivo o mediante la observación de imágenes en dos dimensiones. No obstante, estas últimas plantean mayores dificultades ya que el contexto no provee la misma calidad y cantidad de información. Asimismo los perros utilizan las expresiones emocionales como claves comunicativas para resolver problemas.

Además parecen reconocer diferentes expresiones emocionales de sus congéneres (Maros et al., 2008), rasgo que comparten con sus antepasados, los lobos (Mech \& Boitani, 2003). Los perros conviven hace miles de años con los seres humanos por lo que sería esperable que también desarrollen habilidades para reconocer nuestras emociones y de esta forma regular nuestras interacciones.

No están claros los mecanismos implicados en la adquisición de esta habilidad y en qué medida estos mecanismos son iguales a los que el perro utiliza para detectar otros tipos de claves comunicativas humanas como el señalamiento. Para intentar explicarlos, existen diversos factores tales como la domesticación, el nivel de interacción con los seres humanos a lo largo de la vida, los procesos de aprendizaje espontáneos entre ambas especies, etc., y también se supone que podría existir una predisposición biológica adquirida durante la domesticación para detectar las expresiones emocionales humanas, o quizás los sujetos han aprendido la asociación entre determinadas expresiones emocionales, ciertos patrones de comportamiento y determinadas consecuencias ambientales durante su ontogenia. Lo más plausible es que el interjuego entre factores filogenéticos y ontogenéticos sea el responsable del desarrollo de una habilidad tan compleja como es el reconocimiento de las expresiones emocionales.

Otra pregunta es si los perros comprenden el significado de las expresiones emocionales y si podrían desarrollar una respuesta empática a éstas. Aún no existen evidencias suficientes para afirmar que esto es posible, pero el hallazgo de que los perros se acercan con más frecuencia a personas que simulan llorar podría ser un indicio de respuesta empática o podría tratarse de contagio emocional. También esta conducta podría ser el resultado de un historial de aprendizaje previo en el que los animales fueron reforzados por mostrar conductas de consuelo. En este sentido sería interesante comparar el desempeño de perros de familia y perros alojados por periodos prolongados en refugios caninos donde la interacción con la gente es escasa. Esto permitiría aportar evidencia en favor de la importancia de la historia de aprendizajes en la ontogenia para el desarrollo de esta habilidad.
También queda pendiente indagar más acerca de si existen diferencias entre razas que harían a algunos perros más sensibles que otros a las señales de comportamiento humanos.

Asimismo, de las investigaciones acerca del reconocimiento humano de expresiones emocionales de los perros hasta el momento se podría concluir que las personas pueden discriminar diversas expresiones emocionales y que la experiencia con perros, hace que estas personas tengan un mejor desempeño en el reconocimiento. Sin embargo debemos ser cautelosos al respecto dado que las evidencias son aún escasas, con muestras limitadas y de difícil generalización.

Dado que los perros conviven con seres humanos cumpliendo distintas funciones como animales de compañía y también colaborando en distintos trabajos, tales como las tareas de vigilancia, el control de plagas y la asistencia a personas con discapacidades, entre otros, la comunicación entre ambas especies resulta indispensable. En este contexto y teniendo en cuenta la cantidad de información que brindan las expresiones emocionales, su detección y discriminación es de un valor incalculable para que la comunicación sea efectiva.

\section{Referencias}

Axelsson, E., Ratnakumar, A., Arendt, M. L., Maqbool, K., Webster, M. T., Perloski, M., Liberg, O., Arnemo, J. M., Hedhammar, A., \& Lindblad-Toh, K. (2013). The genomic signature of dog domestication reveals adaptation to a starch-rich diet. Nature, 495, 360364.

Autier-Dérian, D., Deputte, B. L., Chalvet-Monfray, K., Coulon, M., \& Mounier, L. (2013). Visual discrimination of species in dogs (Canis familiaris). Animal Cognition, 16(4), 637-651.

Bandura, A. (1977). Social learning theory. Englewood Cliffs, NJ: Prentice Hall.

Bentosela, M., Barrera, G., Jakovcevic, A., Elgier, A. M., \& Mustaca, A. E. (2008). Effect of reinforcement, reinforcer omission and extinction on a communicative response in domestic dogs. Behavioural Processes, 78(3), 464-469.

Bloom, T. \& Friedman, H. (2013). Classifying dogs' (Canis familiaris) facial expressions from photographs. 
¿Pueden los Perros Domésticos (Canis lupus familiaris) Reconocer Expresiones Emocionales Humanas?

Behavioural Processes, 96, 1-10. doi: 10.1016/j. beproc.2013.02.010

Buttelmann, D. \& Tomasello, M. (2013). Can domestic dogs (Canis familiaris) use referential emotional expressions to locate hidden food? Animal Cognition, 16(1), 137-145. doi: 10.1007/ s10071-012-0560-4.

Custance, D. \& Mayer, J. (2012). Empathic-like responding by domestic dogs (Canis familiaris) to distress in humans: An exploratory study. Animal Cognition, 15(5), 1-31.

Darwin, G. H. (1872). The expression of the emotions in man and animals. London: John Murray.

Davis, S. J. M., Valla, F. R. (1978). Evidence for domestication of the $\operatorname{dog} 12,000$ years ago in the Natufian of Israel. Nature, 276, 608-610.

Ekman, P. \& Friesen, W. V. (1971). Constants across cultures in the face of emotion. Fournal of Personality and Social Psychology, 17(2), 124-129.

Ekman, P. \& Friesen, W. V. (1976). Pictures of facial affect. Palo Alto, CA: Consulting Psychologists Press.

Ekman, P. (1993). Facial expressions of emotion. American Psychologist, 48(4), 384-392.

Elgier, A. M. \& Bentosela, M. (2009). El gesto de señalar: Una llave para la comunicación entre especies. Interdisciplinaria, 26(2), 157-182.

Feiring, C., Lewis, M., \& Starr, M. D. (1984). Indirect effects and infants' reaction to strangers. Developmental Psychology, 20, 485-491.

Fragaszy, D. \& Liu, Q. (2012). Instrumental behavior and problem solving. In N. M. Seel (Ed.), Encyclopedia of the Sciences of Learning. New York, NY: Springer.

Gaunet, F. \& Deputte, B. (2011). Functionally referential and intentional communication in the domestic dog: Effects of spatial and social contexts. Animal Cognition, 14(6), 849-860.

Gazzano, A., Mariti, C., Papi, F., Falaschi, C., Foti, S., \& Ducci, M. (2010). Are domestic dogs able to calm conspecifics by using visual communication? Journal of Veterinary Behavior: Clinical Applications and Research, 5(1), 28-29.
Graham, R., Labar, K. S. (2012). Neurocognitive mechanisms of gaze-expression interactions in face processing and social attention. Neuropsychologia, 50(5), 553-66. doi: 10.1016/j. neuropsychologia.2012.01.019.

Hare, B. \& Tomasello, M. (1999). Domestic dogs (Canis familiaris) use human and conspecific social cues to locate hidden food. Fournal of Comparative Psychology, 113 (2), 173-177.

Hare, B., Rosati, A., Kaminski, J., Brauer, J., Call, J., \& Tomasello, M. (2010). The domestication hypothesis for dogs' skills with human communication: a response to Udell et al. (2008) and Wynne et al. (2008). Animal Behaviour, 79 (2), el-e6. ISSN 00033472 10.1016/j.anbehav.2009.06.031.

Izard, C. E. (1990). Facial expressions and the regulation of emotions. Fournal of Personality and Social Psychology, 58(3), 487-498.

Kim, G., Walden, T. A., \& Knieps, L. J. (2010). Impact and characteristics of positive and fearful emotional messages during infant social referencing. Infant Behavior \& Development, 33(2), 189-195.

Kujala, M. V., Kujala, J., Carlson, S., \& Hari, R. (2012). Dog experts' brains distinguish socially relevant body postures similarly in dogs and humans. PLoS one, 7(6), e39145.

Kundey, S., De Los Reyes, A., Royer, E., Molina, S., Monnier, B., German, R., Coshun, A. (2011). Reputation-like inference in domestic dogs (Canis familiaris). Animal Cognition, 14(2), 291-302.

Lindblad-Toh, K. et al. (2005). Genome sequence, comparative analysis and haplotype structure of the domestic dog. Nature, 438(7069), 803-819.

Maros, K., Pongrácz, P., Bárdos, G., Molnár, C., Faragó, T., \& Miklósi, Á. (2008). Dogs can discriminate barks from different situations. Applied Animal Behaviour Science, 114(1), 159-167.

Marshall-Pescini, S., Passalacqua, C., Miletto Petrazzini, M. E., Valsecchi, P., \& Prato-Previde, E. (2012). Do dogs (Canis lupus familiaris) make counterproductive choices because they are sensitive to human ostensive cues? PLoS one, 7(4), e35437. doi:10.1371/journal. pone.0035437. 
Mech, D. \& Boitani, L. (2003). Wolves. Behavior, ecology and conservation. Chicago: The University of Chicago Press.

Merola, I., Prato-Previde, E., \& Marshall-Pescini, S. (2011). Social referencing in dog-owner dyads? Animal Cognition, 15(2), 175-185.

Merola, I., Prato-Previde, E., \& Marshall-Pescini S. (2012). Dogs' Social Referencing towards Owners and Strangers. PLoS one, 7(10), e47653. doi:10.1371/ journal.pone.0047653.

Miklósi, Á., Kubinyi E., Topál J., Gácsi M., Virányi Z., \& Csányi V. (2003). A simple reason for a big difference: Wolves do not look back at humans but dogs do. Current Biology, 13(9), 763-766.

Miklósi, Á. (2009). Evolutionary approach to communication between humans and dogs. Veterinary Research Communications, 33, 53-59.

Molnár, C., Pongrácz, P., \& Miklósi, Á. (2010). Seeing with ears: Sightless humans' perception of dog bark provides a test for structural rules in vocal communication. Quarterly Fournal of Experimental Psychology, 63(5), 1004-1013.

Mumme, D. L., Fernald, A., \& Herrera, G. (1966). Infants' responses to facial and vocal emotional signals in a social referencing paradigm. Child Development, 67(6), 3219-3237.

Nagasawa, M., Murai, K., Mogi, K., \& Kikusui, T. (2011). Dogs can discriminate human smiling faces from blank expressions. Animal Cognition, 14(4), 525-33.

Nitzschner, M., Melis, A. P., Kaminski, J., \& Tomasello, M. (2012). Dogs (Canis familiaris) evaluate humans on the basis of direct experiences only. PLoS one, 7(10), Special section, 1 .

Pang, J., Kluetsch, C., Zoum, X., Zhang, A., Luo, L., Angleby, H., Ardalan, A., Ekström, C., Sköllermo, A., Lundeberg, J., Matsumura, S., Leitner, T., Zhang, Y., \& Savolainen, P. (2009). mtDNA data indicate a single origin for dogs south of Yangtze River, less than 16,300 years ago, from numerous wolves. Molecular Biology and Evolution, 26(12), 2849-2864.

Pollak, S.D., Cicchetti, D., Hornung, K., \& Reed, A. (2000). Recognizing emotion in faces: Developmental effects of child abuse and neglect. Developmental Psychology, 36, 679-688.
Pongrácz, P., Molnár, G., Miklósi, Á., \& Csányi, V. (2005). Human listeners are able to classify $\operatorname{dog}$ (Canis familiaris) barks recorded in different situations. Fournal of Comparative Psychology, 119(2), 136-144.

Prato-Previde, E., Marshall-Pescini, S., \& Valsecchi, P. (2008). Is your choice my choice? The owners' effect on pet dogs' (Canis lupus familiaris) performance in a food choice task. Animal Cognition, 11(1), 167-174.

Racca, A., Amadei, E., Ligout, S., Guo, K., Meints, K., \& Mills, D. (2010). Discrimination of human and dog faces and inversion responses in domestic dogs (Canis familiaris). Animal Cognition, 13(3), 525-533.

Racca, A., Guo, K., Meints, K., \& Mills, D. S. (2012). Reading faces: Differential lateral gaze bias in processing canine and human facial expressions in dogs and 4-year-old children. PLoS one, 7(4), e36076. doi:10.1371/journal.pone.0036076.

Ramos, D. \& Ades, C. (2012). Two-item sentence comprehension by a dog (Canis familiaris). PLoS one, 7(2), e29689.

Repacholi, B. M. (1998). Infants' use of attentional cues to identify the referent of another person's emotional expression. Developmental Psychology, 34(5), 1017-1025. doi:10.1037/0012-1649.34.5.1017.

Russell, C. L., Bard, K. A., \& Adamson, L. B. (1997). Social referencing by young chimpanzees (Pan troglodytes). Fournal of Comparative Psychology, 111(2), 185-193.

Russell, J. A. (1994). Is there universal recognition of emotion from facial expression? A review of the crosscultural studies. Psychological Bulletin, 115(1), 102-141. doi:10.1037/0033-2909.115.1.102

Russell, Y. I., Call, J., \& Dunbar, R. I. M. (2008). Image scoring in great apes. Behavioural Processes, 78(1), 108111.

Siniscalchi, M., Sasso, R., Pepe, A. M., Vallortigara, G., \& Quaranta, A. (2010). Dogs turn left to emotional stimuli. Behavioural Brain Research, 208(5), 516-521.

Siniscalchi, M., Quaranta, A., \& Rogers, L. J. (2008). Hemispheric specialization in dogs for processing different acoustic stimuli. PLoS one, 3(10), e3349. doi:10.1371/journal.pone.0003349. 


\section{¿Pueden los Perros Domésticos (Canis lupus familiaris) Reconocer Expresiones Emocionales Humanas?}

Sorce, J. F., Emde, R. N., Campos, J., \& Klinnert, M. (1985). Maternal emotional signaling: Its effect on the visual cliff behaviour of 1 year olds. Developmental Psychology, 21(1), 195-200.

Stetina, B. U.,Turnerb, K., Burgerb, E., Glenkc, L. M., McElheneyb, J. C., Handlosd, U., Kothgassnera, O. D. (2011). Learning emotion recognition from canines? Two for the road. Fournal of Veterinary Behaviour: Clinical Applications and Research, 6(2), 108-114.

Tami, G. \& Gallagher, A. (2009). Description of the behaviour of domestic dog (Canis familiaris) by experienced and inexperienced people. Applied Animal Behaviour Science, 120(3-4), 159-169.

Topál, J., Gergely, G., Erdöhegyi, A., Csibra, G., \& Miklósi, Á. (2009). Differential sensitivity to human communication in dogs, wolves, and human infants. Science, 325(5945), 1269-1272.

Topál, J., Gergely, G., Erdöhegyi, A., Csibra, G., \& Miklósi, Á., (2008). Infants' perseverative search errors are induced by pragmatic misinterpretation. Science, 321(5897), 1831-1834.

Udell, M.A.R., Dorey, N. R., Wynne, G. D. L. (2010). What did domestication do to dogs? A new account of dogs' sensitivity to human actions. Biological Reviews, 85(2), 327-345.

Vaish, A. \& Striano, T. (2004). Is visual reference necessary? Contributions of facial versus vocal cues in 12-month- olds' social referencing behavior. Developmental Science, 7(3), 261-269.

Vas, J., Topál, J., Gácsi, M., Miklósi, Á., \& Csányi, V. (2005). A friend or an enemy? Dogs' reaction to an unfamiliar person showing behavioural cues of threat and friendliness at different times. Applied Animal Behaviour Science, 94(1-2), 99-115.

Vila, C., Savolainen, P., Maldonado, J. E., Amorim, I. R., Rice, J. E., Honeycutt, R. L., Crandall, K. A., Lundeberg, J., \& Wayne, R. K. (1997). Multiple and ancient origins of the domestic dog. Science, 276(5319), 1687-1689.

Virányi, Z., Topál, J., Gácsi, M., Miklósi, Á., \& Csányi, V. (2004). Dogs respond appropriately to cues of humans' attentional focus. Behavioural Processes, 66(2), 161-172.

Virányi, Z., Gácsi, M., Kubinyi, E., Topál, J., Belényi, B., Ujfalussy, D., \& Miklósi, Á. (2008). Comprehension of human pointing gestures in young human-reared wolves (Canis lupus) and dogs (Canis familiaris). Animal Cognition, 11(3), 373-387.

Vitulli, W. F. (2006). Attitudes toward empathy in domestic dogs and cats. Psychological Reports, 99(3), 981-991.

Wan, M., Bolger, N., Champagne, F.A. (2012). Human perception of fear in dogs varies according to experience with dogs. PLoS one, 7(12), e51775. doi:10.1371/journal.pone.0051775. 